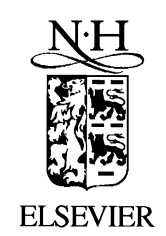

\title{
Morphological instability due to double diffusive convection in directional solidification: the pit formation
}

\author{
C.W. Lan*, C.Y. Tu \\ Department of Chemical Engineering, National Taiwan University, Taipei, Taiwan 10617, ROC
}

Received 14 July 2000; accepted 20 September 2000

Communicated by D.T.J. Hurle

\begin{abstract}
Deep interface depression or "pit formation", as a result of solute accumulation, due to double-diffusive convection in the directional solidification of succinonitrile ( $\mathrm{SCN}$ ) containing ethanol in an ampoule is investigated by a fully nonlinear numerical simulation. The calculated results are consistent with previous observations (Schaefer and Coriell, Metal. Trans. 15A (1984) 2109), and the instability margin falls between the convective and morphological boundaries at a low growth rate. For a high growth rate, the global interface depression becomes deep due to significant release of the heat of fusion; in this case, the critical concentration can be lower than the convective value. Near the instability margin, the pit forms at the center of the interface and is soon followed by constitutional supercooling. Also, the pit shape is affected significantly by the convective solute transport and thus the flow structures. Such pit formation, results from the nonlinear coupling of double-diffusive convection and the interface deformation, and although differs from the traditional mechanisms, it could be an important route to interface breakdown. (C) 2000 Published by Elsevier Science B.V.
\end{abstract}

PACS: 44.25. + f; 47.27.Te; 81.10.Fq; 02.60.Cb; 02.70.Fj

Keywords: Morphology; Instability; Double-diffusive convection; Directional solidification; Pit formation

\section{Introduction}

Since the pioneering work by Mullins and Sekerka [1], the morphological instability of a crystal/melt interface during solidification of an alloy has been extensively studied. The effects of convection have also been investigated (see e.g., Refs. [2,3]). Coriell et al. [2] and Hurle et al. [3]

\footnotetext{
* Corresponding author. Tel./fax: + 886-2-2363-3917.
}

E-mail address: lan@ruby.che.ntu.edu.tw (C.W. Lan). first realized the possibility of coupling of the morphological and convective instabilities. For a thermally stable configuration, through a linear stability analysis for a flat interface, they found that the convective mode, due to the solutal flow (with the lighter solute being rejected from the growth interface), occurs at low-wave-number (or large scale) and the high-wavenumber mode (or small scale) is the reason for the morphological instability proposed by Mullins and Sekerka [1]. They could be loosely or strongly coupled depending on the wavenumber of the onset of their instability. An oscillatory mode was also found by Coriell et al. 
[2], which was believed to be from the strong coupling of both modes, though it was not found in their experiment. For this unstable solutal configuration, Young and Davis [4] also showed that the morphological instability can be retarded by buoyancy below the critical Rayleigh number. Thermally induced convection is also important in the morphological instability. Coriell and McFadden [5] found that the thermal convection alone can promote the instability, and instability starts at a much longer wavelength for tin-containing lead, and the larger thermal conductivity of the solid phase than the melt plays an important role; if the melt had a larger thermal conductivity, the stability can be enhanced. The rejected lead (heavier) during solidification can stabilize the interface. Interestingly, their work in conjunction with the convective instability found earlier [2] indicates that the most dangerous mode (with a much smaller wavenumber or longer wavelength) may appear at a lower solute concentration or a lower solidification rate. More importantly, due to the small wavenumber, the interfacial energy has little effect on the morphological deformation. Therefore, the convective instability as well as the induced morphological instability (may be in a small scale) is of practical importance and has been paid much attention since then (see e.g., Refs. [5-7]). A detailed review of related studies can be found in a review work by Davis [6].

Unfortunately, for directional solidification in an ampoule, using the Bridgman or other similar configurations, the situation is not as simple as the previous linear analyses, which require an infinite domain. Due to the ampoule, the heat of fusion released during solidification can cause interface deformation, even with the same melt and crystal thermal conductivities. Such a deformation produces radial thermal gradients, and as a result, thermal convection is induced. This breaks down the basic assumptions of the previous linear analyses, where the wall effect was ignored and no flow was assumed for the basic state. Also, the supercritical bifurcation of the solutal flow becomes imperfect, and the onset point of the solutal flow does not exist. In other words, the thermal convection predominates in the melt initially. Therefore, in reality the applications of the so-called convective instability due to buoyancy become limited. In fact, under such an imperfect condition, the interface is deformed, and thus deviated from the flat one for a basic state; however, the small-scale interfacial instability may not appear. Nevertheless, the convection-induced morphological instability leading to the cellular growth is still believed to be important, and some interesting observations have been reported. By observing the directional solidification of succinonitrile ( $\mathrm{SCN})$ containing ethanol, Schaefer and Coriell [8] found that there was a pit formation at the center of the interface before the interface breakdown into the cellular mode. They also computed the stability diagram based on a linear stability analysis, but the experimental condition for the pit formation was located between the convective and morphological (or capillary) boundaries. A similar observation for the Bridgman growth of $\mathrm{PbBr}_{2}$, doped with $\mathrm{AgBr}$, was also reported by Singh et al. [9], and their pit development was found even deeper before the small-scale breakdown. Therefore, the pit formation seems to be an important route, may be a short cut, to the interfacial morphological instability. Indeed, as the pit forms, excess solute can accumulate there and facilitate constitutional supercooling.

However, to the authors knowledge, theoretical analysis of pit formation has not yet been carried out. Although Davis [6] gave some discussion on the morphological instability under a stagnation flow through a boundary layer approximation, the analysis was simply qualitative and could not reveal any physical picture observed in experiments. In fact, Coriell and McFadden [5] did clearly mention that the long-wavelength instability, such as the pit formation, requires a fully nonlinear analysis due to the effect of ampoule. Therefore, in this article, we attempt to simulate the process using a fully nonlinear model. The key factors for pit formation and shape are discussed. In addition, the constitutional supercooling occurring during pit formation is further illustrated. In the next section, the mathematical model and its numerical solution are briefly described. Section 3 is devoted to discussion, followed by conclusions in Section 4. 


\section{Model and numerical solution}

The schematic of the directional solidification configuration used in this study is depicted in Fig. 1. The furnace is described by an effective heating profile $T_{\mathrm{a}}(z, t)$, which is assumed linear with a thermal gradient $G$. Both the pseudo-steady state and fully time-dependent (growth dynamic) models are considered. For the pseudo-steady-state calculations, the thermal profile is kept stationary, while the ampoule is moving downward at the speed $U_{\mathrm{a}}$. For the dynamic simulation, the crystal growth starts from a stationary state by moving the thermal profile upward at the speed $U_{\mathrm{h}}$. Furthermore, the system is assumed to be axisymmetric, and the initial dopant distribution in the melt is assumed uniform at $C_{0}$. The flow, temperature, and solute fields, as well as the growth front (the melt/crystal interface, $\left.h_{\mathrm{c}}(r, t)\right)$, are represented in a cylindrical coordinate system $(r, z)$.

The melt is assumed incompressible and Newtonian, while the flow is laminar. The Boussinesq approximation is also adopted. If the stream function $\psi$ and vorticity $\omega$ are defined in terms of radial $(u)$ and axial $(v)$ velocities as

$u=-\frac{1}{\rho_{\mathrm{m}} r} \frac{\partial \psi}{\partial z}, \quad v=\frac{1}{\rho_{\mathrm{m}} r} \frac{\partial \psi}{\partial r}$

and

$\omega=\frac{\partial u}{\partial z}-\frac{\partial v}{\partial r}$

the governing equations for vorticity, stream function, temperature $(T)$, and solute concentration $(C)$ in the conservative-law form can be written as follows:

Equation of motion:

$$
\begin{gathered}
-\rho_{\mathrm{m}} \frac{\partial \omega}{\partial t}+\frac{\partial}{\partial r}\left(\frac{\omega}{r} \frac{\partial \psi}{\partial z}\right)-\frac{\partial}{\partial z}\left(\frac{\omega}{r} \frac{\partial \psi}{\partial r}\right) \\
+\frac{\partial}{\partial r}\left(\frac{1}{r} \frac{\partial}{\partial r}\left(\mu_{\mathrm{m}} r \omega\right)\right)+\frac{\partial}{\partial z}\left(\frac{1}{r} \frac{\partial}{\partial z}\left(\mu_{\mathrm{m}} r \omega\right)\right) \\
-\rho_{\mathrm{m}} \beta_{\mathrm{T}} g \frac{\partial T}{\partial r}+\rho_{\mathrm{m}} \beta_{\mathrm{S}} g \frac{\partial C}{\partial r}=0 .
\end{gathered}
$$

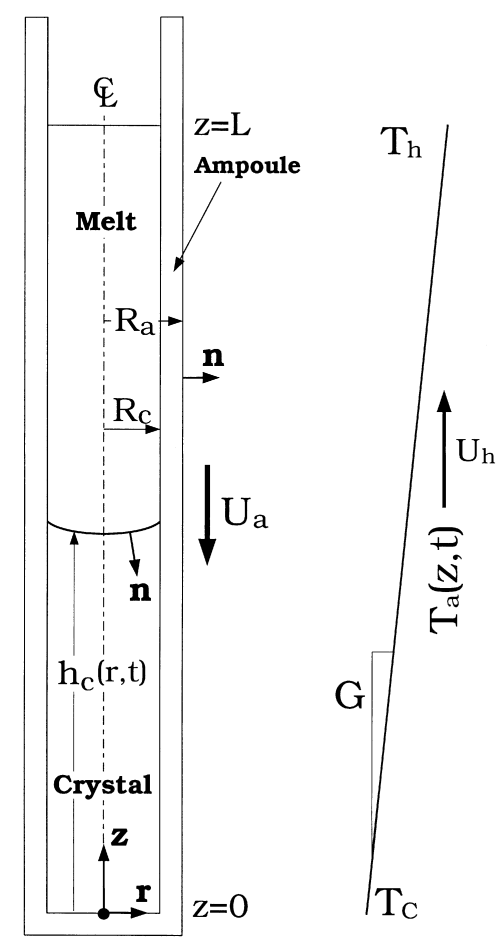

Fig. 1. Schematic sketch of directional solidification in an ampoule.

\section{Stream function equation:}

$$
\frac{\partial}{\partial z}\left(\frac{1}{\rho_{\mathrm{m}} r} \frac{\partial \psi}{\partial z}\right)+\frac{\partial}{\partial r}\left(\frac{1}{\rho_{\mathrm{m}} r} \frac{\partial \psi}{\partial r}\right)+\omega=0 .
$$

Energy equation:

$$
\begin{aligned}
- & \rho_{i} C_{p i} r \frac{\partial T}{\partial t}-\frac{\partial}{\partial r}\left(\rho_{i} C_{p i} r u T\right)-\frac{\partial}{\partial z}\left(\rho_{i} C_{p i} r v T\right) \\
+ & \frac{\partial}{\partial z}\left(r k_{i} \frac{\partial T}{\partial z}\right)+\frac{\partial}{\partial r}\left(r k_{i} \frac{\partial T}{\partial r}\right)=0, \quad i=(\mathrm{m}, \mathrm{c}, \mathrm{a}) .
\end{aligned}
$$

Solute equation:

$$
\begin{aligned}
- & \frac{\partial C}{\partial t}-\frac{\partial}{\partial r}(r u C)-\frac{\partial}{\partial z}(r v C) \\
& +\frac{\partial}{\partial z}\left(r D_{i} \frac{\partial C}{\partial z}\right)+\frac{\partial}{\partial r}\left(r D_{i} \frac{\partial C}{\partial r}\right)=0, \quad i=(\mathrm{m}, \mathrm{c}, \mathrm{a}),
\end{aligned}
$$


where $\mu_{\mathrm{m}}$ is the melt viscosity. Also, $\rho_{i}$ is the density, $C_{p i}$ the specific heat, and $k_{i}$ the thermal conductivity of phase $i$; $i$ represents the melt $(\mathrm{m})$, the crystal (c), or the ampoule (a). In addition, $g$ is the gravitational acceleration, $\beta_{\mathrm{T}}$ and $\beta_{\mathrm{S}}$ are the thermal and solutal expansion coefficients, respectively, and $D_{i}$ is the solute diffusivity in the phase $i$; $i=(\mathrm{m}, \mathrm{c}, \mathrm{a})$.

To solve the above equations, boundary conditions need to be specified. Most boundary conditions can be found elsewhere $[10,11]$. In brief, the no-slip boundary condition for velocities is used at solid boundaries, and the vorticity boundary conditions can be derived from there. The upper melt interface is assumed stress free. For pseudo-steadystate calculations, the solute boundary conditions can also be found in Ref. [11]. For time-dependent calculations, the boundary conditions for the solute at the upper and melt/crystal interfaces are obtained through the balance there:

$$
-\left.\left(\boldsymbol{n} \cdot D_{\mathrm{m}} \nabla C\right)\right|_{z=L}=0
$$

and

$$
\begin{array}{r}
-\left.\left(\boldsymbol{n} \cdot D_{\mathrm{m}} \nabla C\right)\right|_{\mathrm{m}}+\left.\left(\boldsymbol{n} \cdot D_{\mathrm{c}} \nabla C\right)\right|_{\mathrm{c}} \\
-\left.(1-K) C \frac{\partial h_{\mathrm{c}}}{\partial t}\left(\boldsymbol{n} \cdot \boldsymbol{e}_{z}\right)\right|_{\mathrm{c}}=0,
\end{array}
$$

where $\boldsymbol{n}$ is the unit normal vector at the interface pointing outwards, $\boldsymbol{e}_{z}$ the unit vector in the axial direction, and $K$ the segregation coefficient at small solute concentration obtained from the phase diagram; $K \equiv C_{\mathrm{c}} / C$ at the growth interface, where $C_{\mathrm{c}}$ is the solute concentration on the crystal side. The zero flux condition is used at the centerline and the melt/ampoule interface; the solid-state diffusion of the solute in the ampoule is neglected.

Moreover, the liquidus temperature at the interface is a function of the solute concentration and the local curvature (the Gibbs-Thomson equation) as

$$
T_{\mathrm{m}}=T_{\mathrm{m} 0}(1-\Gamma \gamma)+m C,
$$

where $\Gamma$ is the capillary constant, $\gamma$ the mean curvature of the interface, and $m$ the slope of the liquidus line. The contribution of the capillary term is small for the macroscopic morphology. The results ob- tained with and without this term are almost the same.

Finally, for convenience, at the interface the onset condition for constitutional supercooling is characterized by $\Delta G$, which is defined as the difference of the actual $\left(G_{\mathrm{T}}\right)$ and the liquidus $\left(G_{\mathrm{L}}\right)$ temperature gradients at the centerline:

$\Delta G=G_{\mathrm{T}}-G_{\mathrm{L}}$.

Constitutional supercooling occurs when $\Delta G<0$. The liquidus temperature gradient $G_{\mathrm{L}}$ is calculated directly from the concentration gradient $G_{\mathrm{C}}$, i.e., $G_{\mathrm{L}}=m G_{\mathrm{C}}$. Due to the interfacial energy, which usually stabilizes the interface, the condition of $\Delta G<0$ does not guarantee interface instability. According to the pseudo-steady-state analysis of Mullins and Sekerka [1], there is a stability function $f\left(\lambda, m, K, U_{\mathrm{a}}, D_{\mathrm{m}}\right)$ to counterbalance the supercooling before the most unstable cellular mode (i.e., with the most favorable wavelength) to develop; $\lambda$ in $f$ is the wavelength of the unstable interface. In other words, the unstable growth occurs at $\Delta G+f<0$. Another form taken from Ref. [12] is to use a function $\delta(A, K)$, and the stability is retained for

$G_{\mathrm{T}}-G_{\mathrm{L}} \delta(A, K)>0$,

where $A \equiv\left(K^{2} /(1-K)\right)\left(\Gamma U_{\mathrm{a}} / D_{\mathrm{m}}\right)\left(T_{\mathrm{m} 0} /\left(-m C_{0}\right)\right)$; $U_{\mathrm{a}}$ is set to be the steady growth speed. For a constant segregation coefficient $K$, the greater the $A$ value, the lower the $\delta$ value (also see Fig. 15.11 in Ref. [12]), and therefore the higher the interface stability. For example, the $A$ value for $\mathrm{PbBr}_{2} / \mathrm{AgBr}$ system $\left(\approx 10^{-4}\right)$ in Ref. [9] is two orders higher than that for $\mathrm{SCN} /$ ethanol $\left(\approx 10^{-6}\right)$; therefore, $\delta$ is about one for $\mathrm{SCN} /$ ethanol and about 0.91 for $\mathrm{PbBr}_{2} / \mathrm{AgBr}$. In other words, the $\mathrm{PbBr}_{2} / \mathrm{AgBr}$ system allows more supercooling before the capillary breakdown; its much higher $K$ value $(0.16$ versus 0.044 ) also plays a crucial role in lowering the $\delta$ value. In fact, there are other similar criteria being proposed $[13,14]$, but it may be safe, especially for $\mathrm{SCN} / \mathrm{ethanol}$, to take $f=0$ or $\delta=1$ here as a lower bound of the instability. Indeed, through the stability function $f$ or $\delta$, one may bridge the present simulation to the prediction of the possible breakdown mode. Unfortunately, an exact form of the function is not available for the configuration here, 
and the solute field near the growth front can only be solved numerically. Therefore, for simplicity, in this study we have taken the constitutional supercooling as the instability criterion.

The governing equations given above and their associated boundary conditions can only be solved numerically. We have developed efficient finite volume methods using Newton's method and the DASPK solver $[10,11,15,16]$ for solving these equations. In brief, the finite volume method is to integrate the conservation equations over control volumes, which are defined by the mesh. After the Gauss theorem is applied, the flux balance equations are obtained; meanwhile, the boundary conditions can be imposed. The nonlinear algebraic (or ordinary differential) equations can then be solved globally by Newton's iteration. The beauty of Newton's iteration is that an additional scheme for the interface calculation is not necessary; all the variables are treated in the same way and they converge simultaneously. Furthermore, bifurcation analyses through continuation and eigenvalue analyses [17] can be implemented easily. Furthermore, excellent global solute conservation can be easily achieved even with a coarse mesh, and this is a great advantage of the finite volume method. Detailed description of the numerical method can be found elsewhere [10].

\section{Results and discussion}

The SCN/ethanol system studied by Schaefer and Coriell [8] is considered here. The physical properties and some input parameters are listed in Table 1 for reference. In Table 1, the solute diffusivity and the solutal expansion coefficient are estimated; their effects will be discussed shortly. To focus our discussion, only the thermal gradient of $10 \mathrm{~K} \mathrm{~cm}^{-1}$ is presented here. The results for different thermal gradients are similar.

Although the computer code used here has been tested extensively and benchmarked with a twodimensional finite element [10] and a three-dimensional primitive-variable model [15], care must be taken to avoid possible numerical artifacts. Therefore, before presenting our calculated results, we have performed several mesh refinements. Fig. 2 shows three meshes and their calculated flow and solute fields for a condition near the onset of constitutional supercooling $\left(U_{\mathrm{a}}=1.66 \times 10^{-4} \mathrm{~cm} \mathrm{~s}^{-1}\right.$; $\left.C_{0}=2.6 \times 10^{-3} \mathrm{wt} \%\right)$ during the pit formation. The converged meshes are shown in Fig. 2a. There are $33 \times 66$ finite volumes in the melt for mesh M1, $63 \times 96$ for M2, and $73 \times 136$ for mesh M3. As shown from the calculated results in Fig. 2b, the effect of meshes is not significant; the calculated pit shapes are very close to one another. Since mesh M2 is satisfactory, to balance the accuracy and the computational cost, it is used for the rest of calculations in the following sections.

The effect of the growth rate is illustrated first in Fig. 3 based on the pseudo-steady-state calculations. The results for zero gravity (absence of the buoyancy force) are illustrated first from Figs. 3a-c. As shown in Fig. 3a, without growth $\left(U_{\mathrm{a}}=0\right)$, there is no flow and the growth interface is almost flat. Increasing the growth rate to $0.6 \times 10^{-4} \mathrm{~cm} \mathrm{~s}^{-1}$ leads to a solute buildup in front of the growth interface (Fig. 2b); the straight streamlines are due to the moving ampoule. The interface shape becomes slightly concave due to the heat of fusion released. As a result, some radial solute segregation exists at the growth front. As the growth speed is increased to $2.51 \times 10^{-4} \mathrm{~cm} \mathrm{~s}^{-1}$, as shown in Fig. $3 \mathrm{c}$, the interface concavity further increases. The ethanol buildup increases significantly as well leading to a thin solute boundary layer near the interface. The radial solute segregation also increases due to the more deformed interface shape. Meanwhile, at this growth speed, constitutional supercooling appears. However, the interface is in general smooth and no local depression (pit) is found.

With convection at normal gravity, as shown from Figs. 3d-f, the flow and solute fields become quite different. Without growth, as illustrated in Fig. 3d, an extremely weak melt convection is induced, which is due to the tiny interface deflection caused by the small difference in the thermal properties of the melt and the crystal. Because the growth rate is zero, no segregation is induced. With a finite growth rate $\left(U_{\mathrm{a}}=-0.6 \times 10^{-4} \mathrm{~cm} \mathrm{~s}^{-1}\right)$, as shown in Fig. 3e, the interface deflection increases; the melt convection is enhanced as well. Significant solute segregation is also observed, which can be 
Table 1

Physical properties and some input parameters [8]

\section{SCN \\ Density of solid \\ Density of melt \\ Melting point \\ Heat of fusion \\ Thermal conductivity of solid \\ Thermal conductivity of melt \\ Specific heat of solid \\ Specific heat of melt \\ Thermal expansion coefficient \\ Liquidus slope \\ Solutal expansion coefficient \\ Segregation coefficient \\ Diffusion coefficient of ethanol \\ In the melt \\ In the crystal \\ In the ampoule \\ Capillary constant}

Pyrex tube

Density

Thermal conductivity

Specific heat

Other input parameters

Ampoule length for simulation

Crystal radius

Ampoule radius

Heater thermal gradient

Hot-zone temperature

Cold-zone temperature

Heat transfer coefficient

$$
\begin{aligned}
& \rho_{\mathrm{c}}=\rho_{\mathrm{f}}=1.016 \mathrm{~g} \mathrm{~cm}^{-3} \\
& \rho_{\mathrm{m}}=0.988 \mathrm{~g} \mathrm{~cm}^{-3} \\
& T_{\mathrm{m}}=331.5 \mathrm{~K} \\
& \Delta H=46.5 \mathrm{~J} \mathrm{~g}^{-1} \\
& k_{\mathrm{c}}=2.25 \times 10^{-3} \mathrm{~W} \mathrm{~cm}^{-1}{ }^{\circ} \mathrm{C}^{-1} \\
& k_{\mathrm{m}}=2.24 \times 10^{-3} \mathrm{~W} \mathrm{~cm}^{-1}{ }^{\circ} \mathrm{C}^{-1} \\
& C_{P_{\mathrm{c}}}=1.955 \mathrm{~J} \mathrm{~g}^{-1{ }^{\circ}} \mathrm{C}_{-1} \\
& C_{p_{\mathrm{m}}}=2.0 \mathrm{~J} \mathrm{~g} \mathrm{~g}^{-1}{ }^{\circ} \mathrm{C}^{-1} \\
& \beta_{\mathrm{T}}=8.1 \times 10^{-4} \mathrm{~K}^{-1} \\
& m=-3.6 \mathrm{Kwt}^{\circ} \%^{-1} \\
& \beta_{\mathrm{S}}=-2.53 \times 10^{-3} \mathrm{wt}^{-1} \text { (estimated) } \\
& K=0.044
\end{aligned}
$$

$$
\begin{aligned}
& \rho_{\mathrm{a}}=2.2 \mathrm{~g} \mathrm{~cm}^{-3} \\
& k_{\mathrm{a}}=0.035 \mathrm{~W} \mathrm{~cm}^{-1}{ }^{\circ} \mathrm{C}^{-1} \\
& C_{p_{\mathrm{a}}}=0.188 \mathrm{~J} \mathrm{~g} \mathrm{~g}^{-1}{ }^{\circ} \mathrm{C}^{-1}
\end{aligned}
$$

$$
\begin{aligned}
& L=14 \mathrm{~cm} \\
& R_{\mathrm{c}}=0.85 \mathrm{~cm} \\
& R_{\mathrm{a}}=1.05 \mathrm{~cm}^{-1} \\
& G=10 \mathrm{~K} \mathrm{~cm}^{-1} \\
& T_{\mathrm{h}}=401.5 \mathrm{~K} \\
& T_{\mathrm{c}}=261.5 \mathrm{~K} \\
& h=1.6 \times 10^{-2} \mathrm{~W} \mathrm{~cm}^{-2 \circ} \mathrm{C}^{-1}
\end{aligned}
$$

seen from the highly distorted solute field. Interestingly, as the growth rate is further increased to near $1.68 \times 10^{-4} \mathrm{~cm} \mathrm{~s}^{-1}$, as shown in Fig. 3f, besides further depression of the interface, a clear pit forms at the center. The pit depth increases with the growth rate, but it is soon followed by constitutional supercooling $(\Delta G<0)$ at $U_{\mathrm{a}}=$ $1.683 \times 10^{-4} \mathrm{cms}^{-1}$. As shown, much ethanol is accumulated inside the pit.

The corresponding radial solute segregations in the grown crystal for Figs. 3d-f are further illustrated in Fig. 4; $C_{\mathrm{c}}=K C$, where $C$ is the solute concentration at the melt side. Clearly, the pit re- sults in severe radial solute segregation. Again, this is due to the solute accumulation there. Therefore, from Fig. 3f, it is clear that the ethanol rejected from the interface during growth is swept to the centerline by convection. The significant local solute accumulation and thus the much lower liquidus temperature facilitate the pit formation. As the pit is formed, the solute accumulation inside the pit further increases, and the significant buildup of the ethanol causes the supercooling to occur at a much lower growth speed. Indeed, as we return to the pseudo-steady analysis of Mullins and Sekerka [1], the interface solute concentration being $C_{0} / K$ (or 

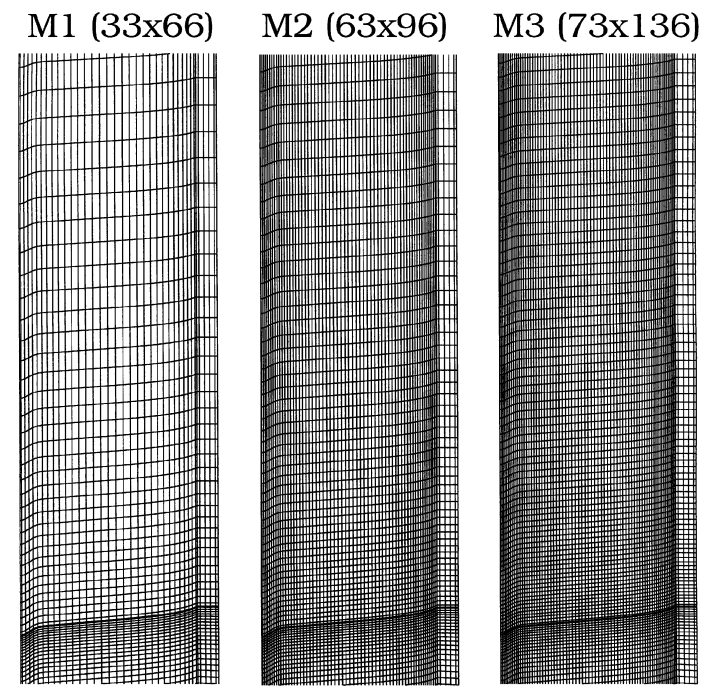

(a)
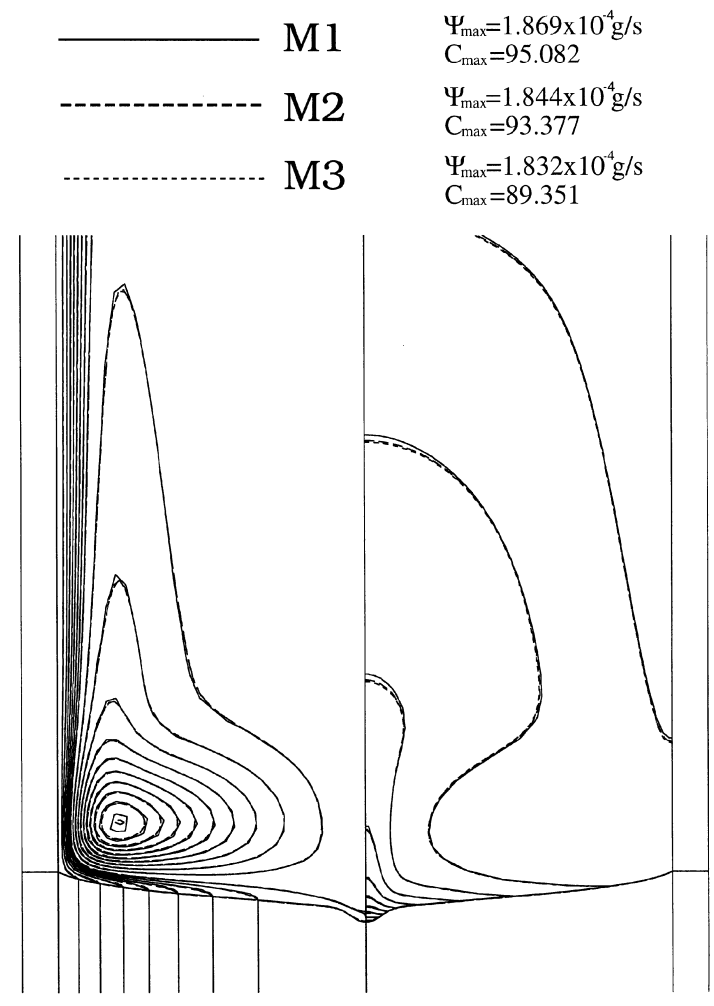

(b)

Fig. 2. Mesh refinements: (a) numerical meshes; (b) calculated flow (left) and solute (right) fields and the interface shape at $U_{\mathrm{a}}=$ $-1.66 \times 10^{-4} \mathrm{~cm} \mathrm{~s}^{-1}$; the concentration is normalized by $C_{0}$ $\left(2.6 \times 10^{-3} \mathrm{wt} \%\right)$.
$\left.C_{\mathrm{c}} / C_{0}=1\right)$ does not hold here. Due to the radial solute segregation, as shown in Fig. 4, the ethanol concentration at the centerline is more than four times higher than the average value $\left(C_{\mathrm{c}} / C_{0}=1\right)$. Therefore, there is no doubt that the supercooling and further the interface breakdown should occur first at the interface center and much earlier than the prediction, which in turn accelerates the instability. In fact, this is what was observed by Schaefer and Coriell [8] and Singh et al. [9]. They tried to explain the pit formation by the convective instability due to the onset of solutal flow. Unfortunately, this mechanism is not quite true. With the interface deflection and thus the radial thermal gradients, thermal convection is induced. Even with the melt flow, the growth rate also needs to be high enough to form the pit before supercooling in this case.

To further illustrate the pit formation, a timedependent calculation is further carried out with $U_{\mathrm{h}}=2.5 \times 10^{-4} \mathrm{~cm} \mathrm{~s}^{-1}$; the result of Fig. $3 \mathrm{~d}$ is used as the initial condition. Also, we have used a higher heater speed here in order to see a supercooling in a reasonable (short) growth distance. As shown in Fig. 5, the thermal gradient difference $(\Delta G$ or $G_{\mathrm{T}}-G_{\mathrm{L}}$ ) at the centerline decreases as the growth proceeds. $G_{\mathrm{T}}$ remains about the same during growth, but the gradient of the liquidus temperature $\left(G_{\mathrm{L}}\right)$ increases with time and it is zero at $t=0$. In a recent analytical analysis by Nastac [18], it was shown that $G_{\mathrm{L}}$ was the highest at $t=0$ and decreased to a constant value at the steady state. However, his assumption was not realistic because he set the growth rate to be constant starting from $t=0$ in his derivation. As shown in the upper part of Fig. 5, the growth rate at $r=0$ is zero at the beginning, and it takes time to catch up with the heater moving speed $U_{\mathrm{h}}$.

In Fig. 5a, a small pit forms at about $t \approx 20130 \mathrm{~s}$ right before supercooling. As the growth proceeds further, the growth speed starts to decrease, while the rest of the interface is still moving at the same speed as the heater, and as a result the pit becomes deeper. Meanwhile, the supercooling continues to increase until the local growth rate is significantly reduced to relieve further build up of the ethanol. However, the reduced growth rate also makes the pit even deeper and finally the simulation is forced to stop due to numerical breakdown. Furthermore, 


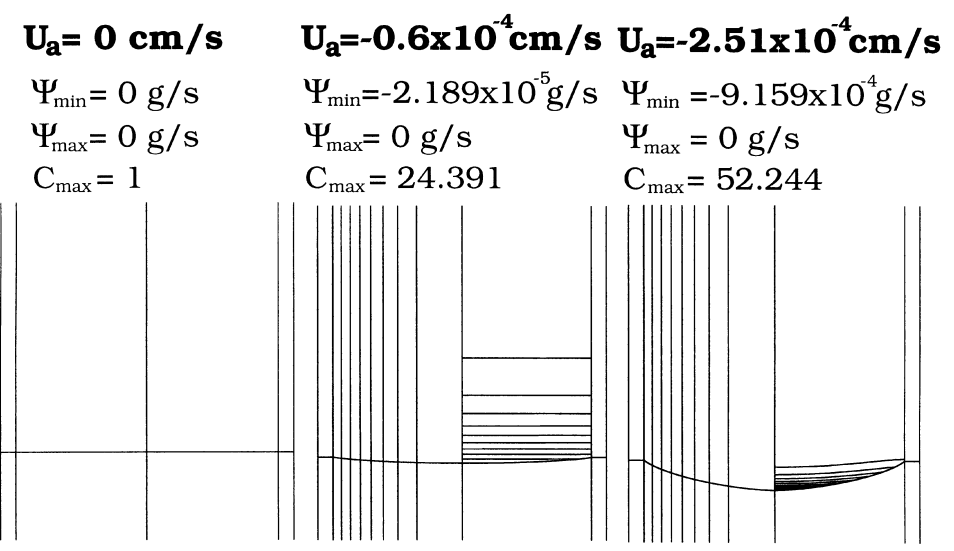

(a)

(b)

(c)

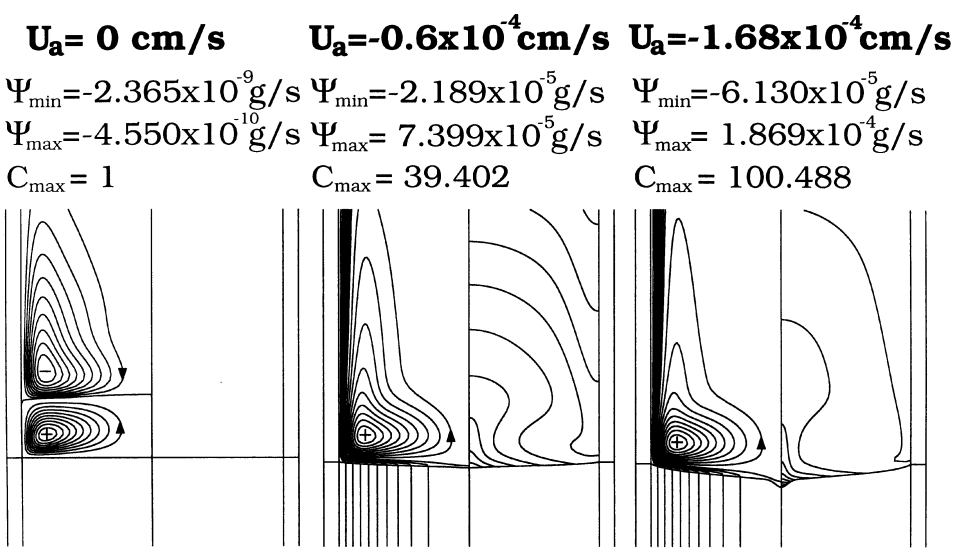

(d)

(e)

(f)

Fig. 3. Effect of growth rate and convection: (a) $U_{\mathrm{a}}=0, g=0$; (b) $U_{\mathrm{a}}=-0.6 \times 10^{-4} \mathrm{~cm} \mathrm{~s}^{-1}, g=0$; (c) $U_{\mathrm{a}}=-2.51 \times 10^{-4} \mathrm{~cm} \mathrm{~s}^{-1}$, $g=0 \quad$ (with supercooling); (d) $U_{\mathrm{a}}=-0 \mathrm{~cm} \mathrm{~s}^{-1}, \quad \mathrm{~g}=980 \mathrm{~cm} \mathrm{~s}^{-2} ; \quad$ (e) $U_{\mathrm{a}}=-0.6 \times 10^{-4} \mathrm{~cm} \mathrm{~s}^{-1}, \quad g=980 \mathrm{~cm} \mathrm{~s}^{-2} ; \quad$ (f) $U_{\mathrm{a}}=$ $-1.68 \times 10^{-4} \mathrm{~cm} \mathrm{~s}^{-1}, g=980 \mathrm{~cm} \mathrm{~s}^{-2}$ (with supercooling); $C_{0}=2.6 \times 10^{-3} \mathrm{wt}^{\mathrm{a}} \mathrm{o}$.

if the interface is stable enough (with a high enough $f$ or small enough $\delta$ value) to sustain the high supercooling at $b$, one may expect to see a deep pit in the experiment. Hence, this might explain the observation of the deep pit (hole) found in the $\mathrm{PbBr}_{2} / \mathrm{AgBr}$ system [9]; as mentioned previously, its $\delta$ value (about 0.91 ) is also much lower than that of the SCN/ethanol system (about one).

Because the directional solidification studied here is a batch process, during crystal growth, the ethanol concentration in the melt continues to increase due to the ethanol rejection from the growth interface $(K=0.044)$. If the melt height is long enough, a pseudo-steady state may be achieved. However, it is still too time consuming to find the pit-formation condition through the time-dependent calculation for a long sample. Instead, the pseudo-steady-state calculations are adopted for the construction of the stability diagram, and a comparison with the previous study is then possible. For a given inlet solute concentration $\left(C_{0}\right)$, one can find a growth rate for the onset of supercooling similar to the work done in Fig. 3. Through a series of calculations, the stability diagram is depicted, as shown in Fig. 6; the results of Schaefer and Coriell [8] are also included for comparison. 


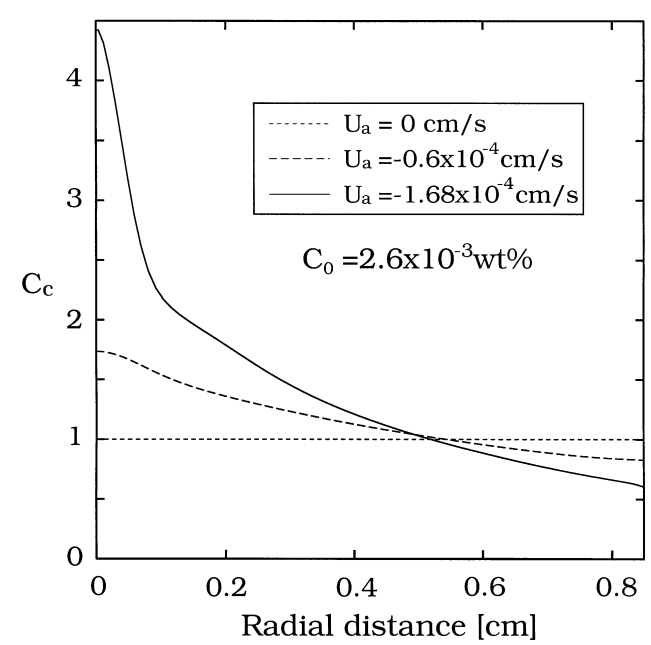

Fig. 4. Radial solute segregation from Figs. 3d-f.

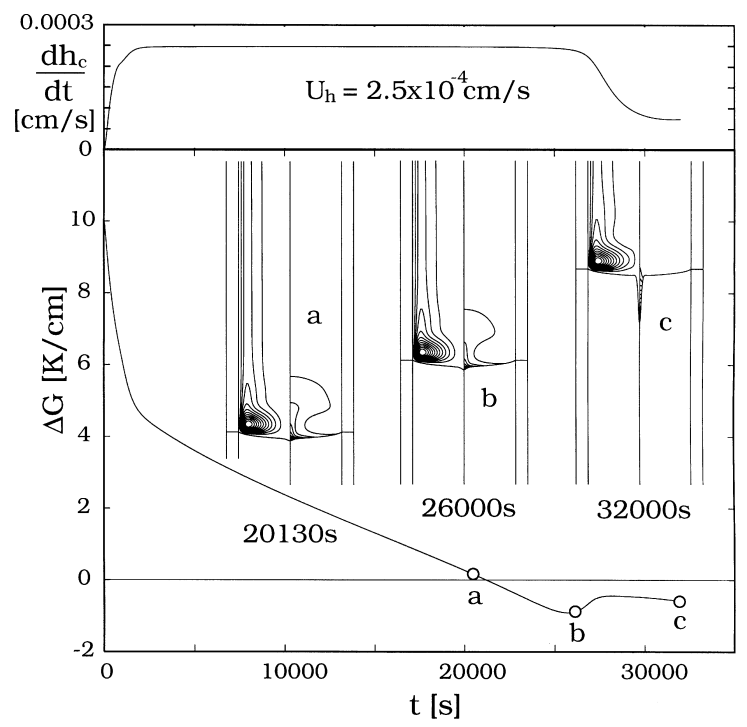

Fig. 5. Time evolution of $\Delta G$ and the growth rate at $r=0$ : (a) the flow and solute fields at $t=20130 \mathrm{~s}$; (b) at $26000 \mathrm{~s}$; (c) at $32000 \mathrm{~s} ; U_{\mathrm{h}}=2.5 \times 10^{-4} \mathrm{~cm} \mathrm{~s}^{-1}$ and $C_{0}=2.6 \times 10^{-3} \mathrm{wt} \%$.

The case without buoyancy flow is also included. As shown, at the lower growth rate, our result lies between two instability boundaries of [8], and is better consistent with the experimental observation. One can also extrapolate our curve to the high-concentration limit, and it is consistent with the capillary one. However, for a growth rate high-

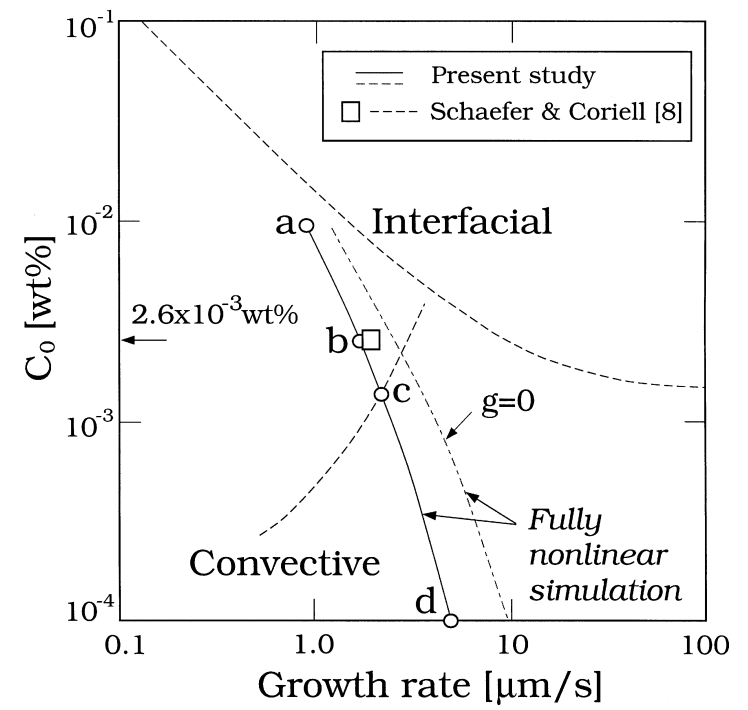

Fig. 6. Stability diagram; the instability of present study is based on $\Delta G<0$.

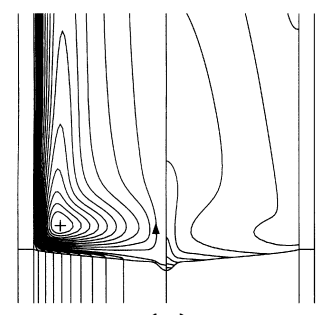

(a)

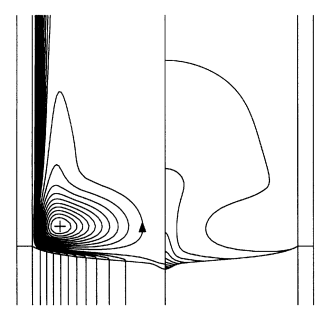

(c)

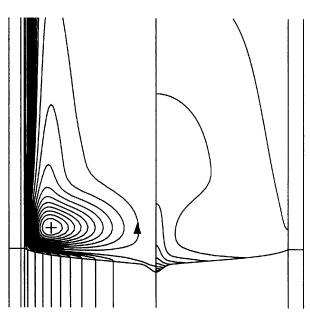

(b)

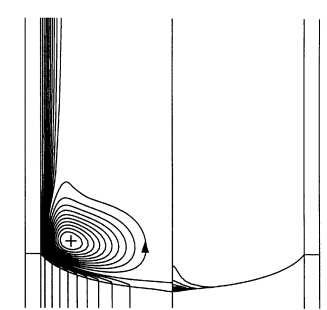

(d)
Fig. 7. Typical flow and solute fields and interface shapes at the instability boundary from Fig. 6: (a) $\Psi_{\min }=-3.284 \times 10^{-5}$, $\Psi_{\max }=5.47 \times 10^{-4}, C_{\max }=51.144$; (b) $\Psi_{\min }=-6.141 \times 10^{-5}$, $\Psi_{\text {max }}=1.869 \times 10^{-4}, \quad C_{\max }=102.596 ;$ (c) $\Psi_{\min }=-7.773 \times$ $10^{-5}, \quad \Psi_{\max }=2.423 \times 10^{-4}, \quad C_{\max }=132.715 ; \quad$ (d) $\Psi_{\min }=$ $-1.788 \times 10^{-4} \mathrm{~g} \mathrm{~s}^{-1}, \Psi_{\max }=5.474 \times 10^{-4} \mathrm{~g} \mathrm{~s}^{-1}, C_{\max }=432.702$.

er than $2.5 \times 10^{-4} \mathrm{~cm} \mathrm{~s}^{-1}$, our critical concentration is lower than the convective one. Some flow and solute fields at the instability boundary are 
further illustrated in Fig. 7. As shown, at higher solute concentration, such as (a)-(c), the pit formation is obvious. On the contrary, with a much lower solute concentration like the case (d), the pit formation is not clear at all. The overall interface deformation in (d) is large, and again this is due to the significant heat of fusion released at the high growth rate. The reduction of $G_{\mathrm{T}}$ due to the heat of fusion also contributes much to the supercooling. The instability boundary for no buoyancy convection, due to the less radial segregation, lies a bit higher indicating that the system is more stable.

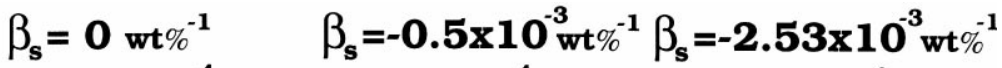

$$
\begin{aligned}
& U_{\mathrm{a}}=-1.4 \times 10^{-4} \mathrm{~cm} / \mathrm{s} \quad U_{\mathrm{a}}=-1.4 \times 10^{-4} \mathrm{~cm} / \mathrm{s} \quad U_{\mathrm{a}}=-1.68 \times 10^{-4} \mathrm{~cm} / \mathrm{s} \\
& \Psi_{\min }=-5.109 \times 10^{-5} \mathrm{~g} / \mathrm{s} \quad \Psi_{\min }=-5.109 \times 10^{-5} \mathrm{~g} / \mathrm{s} \quad \Psi_{\min }=-6.141 \times 10^{-5} \mathrm{~g} / \mathrm{s} \\
& \Psi_{\max }=1.600 \times 10^{-4} \mathrm{~g} / \mathrm{s} \quad \Psi_{\max }=1.597 \times 10^{-4} \mathrm{~g} / \mathrm{s} \quad \Psi_{\max }=1.869 \times 10^{-4} \mathrm{~g} / \mathrm{s} \\
& \mathrm{C}_{\max }=103.758 \quad \mathrm{C}_{\max }=111.788 \quad \mathrm{C}_{\max }=100.488
\end{aligned}
$$

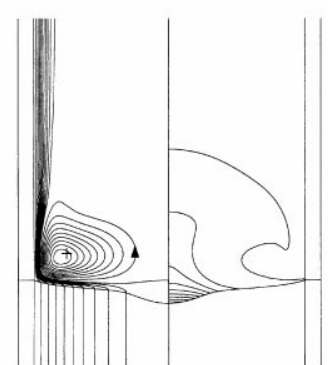

(a)

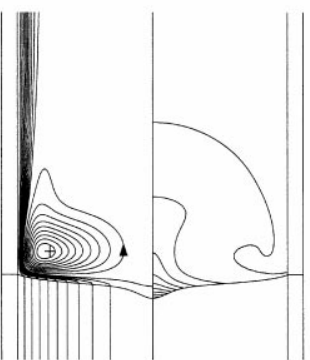

(b)

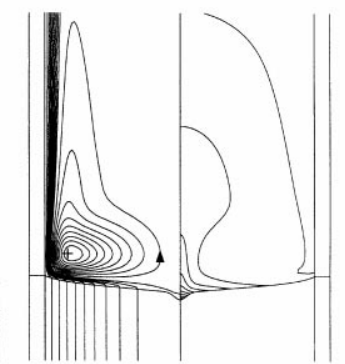

(c)

Fig. 8. Effect of solutal expansion coefficient $\beta_{\mathrm{S}}$ on the flow and solute fields and the pit shape at supercooling: (a) $\beta_{\mathrm{S}}=0 \mathrm{wt} \%{ }^{-1} ;$ (b) $\beta_{\mathrm{S}}=-0.5 \times 10^{-3} \mathrm{wt}^{-1}{ }^{-1}$; (c) $\beta_{\mathrm{S}}=-2.53 \times 10^{-3} \mathrm{wt}^{\mathrm{o}}{ }^{-1} ; C_{0}=2.6 \times 10^{-3} \mathrm{wt}^{\circ}$.

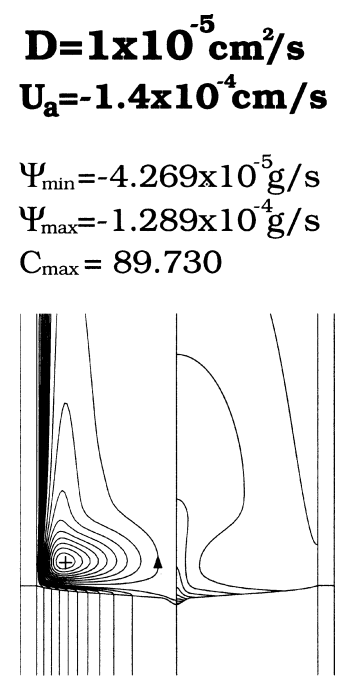

(a)

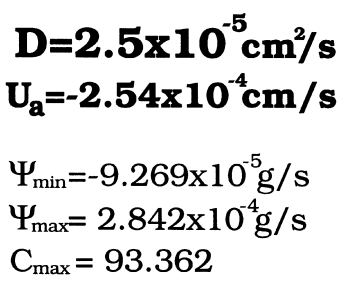

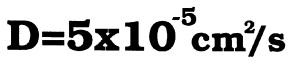
$\mathrm{U}_{\mathrm{a}}=-4.32 \times 10^{-4} \mathrm{~cm} / \mathrm{s}$

$\Psi_{\min }=-1.576 \times 10^{-5} \mathrm{~g} / \mathrm{s}$

$\Psi_{\max }=4.687 \times 10^{-4} \mathrm{~g} / \mathrm{s}$

$\mathrm{C}_{\max }=88.177$

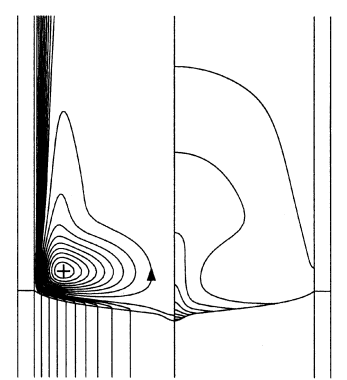

(b)

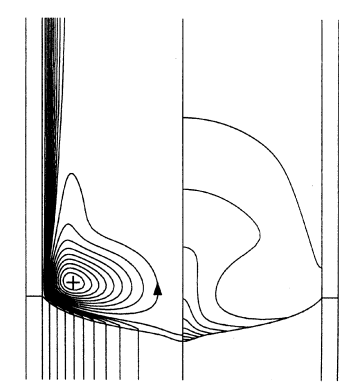

(c)

Fig. 9. Effect of ethanol diffusion coefficient $D_{\mathrm{m}}$ in the melt on the flow and solute fields and the pit shape at supercooling: (a) $D_{\mathrm{m}}=1 \times 10^{-5} \mathrm{~cm}^{2} \mathrm{~s}^{-1}$; (b) $D_{\mathrm{m}}=2.5 \times 10^{-5} \mathrm{~cm}^{2} \mathrm{~s}^{-1}$; (c) $D_{\mathrm{m}}=5 \times 10^{-5} \mathrm{~cm}^{2} \mathrm{~s}^{-1} ; C_{0}=2.6 \times 10^{-3} \mathrm{wt} \%$. 
The pit shape is affected by several factors. Since we do not have accurate values for the solute diffusivity in the melt $\left(D_{\mathrm{m}}\right)$ and the solutal expansion coefficient $\left(\beta_{\mathrm{S}}\right)$, their effects on the pit shape are further illustrated. Fig. 8 shows the calculated results with different $\beta_{\mathrm{S}}$ 's at the critical velocity (i.e., $\Delta G=0)$. As shown, without the solutal convection $\left(\beta_{\mathrm{S}}=0\right)$, the pit shape at supercooling becomes much wider. Increasing the solutal effect makes the pit narrower, as shown in Figs. $8 \mathrm{~b}$ and c, while the critical velocity also increases slightly, i.e., becomes more stable. Because ethanol is lighter than the melt, its accumulation in the pit also enhances the local melt convection due to the unstable configuration leading to a more distorted solute field there. As a result, the pit shape becomes narrower. The value used in Fig. 8 (also Fig. 6), $\beta_{\mathrm{S}}=$ $-2.53 \times 10^{-3} \mathrm{wt}^{-1} \%^{-1}$, is taken from the density measurements from Ref. [8], and it may be a better value to use.

The solute diffusivity has a similar effect. The thickness of the solute boundary layer increases with increasing diffusivity (or with the decreasing Schmidt number Sc, which is the ratio of the melt kinematic viscosity $\left(\mu_{\mathrm{m}} / \rho_{\mathrm{m}}\right)$ and the solute diffusivity $D_{\mathrm{m}}$ ), as shown in Fig. 9. The ethanol accumulation inside the pit also decreases with increasing diffusivity due to faster diffusion. As a result, the constitutional supercooling becomes less likely and occurs at a much higher growth rate, such as the case in Fig. 9c. Again, we believe that the value of $1.5 \times 10^{-5} \mathrm{~cm}^{2} \mathrm{~s}^{-1}$ may be a reasonable value to use for organic systems. In fact, we have found that in Fig. $6, D_{\mathrm{m}} \approx 1.4 \times 10^{-5} \mathrm{~cm}^{2} \mathrm{~s}^{-1}$ gives the best fit to the experimental data.

\section{Conclusions}

The pit formation due to double-diffusive convection in the directional solidification of SCNcontaining ethanol is investigated through a fully nonlinear simulation. The calculated results agree well with the experimental observations although the short-wavelength mode is not considered explicitly in the computation. At high solute concentration, the pit formation at the center of the interface becomes obvious before supercooling. As it appears, the significant solute accumulation there accelerates the constitutional supercooling leading to the interface breakdown. From the transient calculation, we also illustrate that a deep pit may be possible if the interface stability is high enough to sustain the supercooling, which might explain the deep hole formation found in the $\mathrm{PbBr}_{2} / \mathrm{AgBr}$ system. At low solute concentration, a higher growth rate is required for supercooling, but the pit formation is not obvious. The instability and the pit morphology are further influenced by the local flow and solute diffusion. Although the present calculations are mainly for the long-wavelength instability, the pit formation mechanism due to buoyancy convection is believed to be a short cut to interfacial instability during the directional solidification of a concentrated alloy.

\section{Acknowledgements}

This work was sponsored by the National Science Council and the National Center for HighPerformance Computing of the Republic of China under Grant No. NSC89-2214-E002-040. Some physical data provided by Dr. Coriell are deeply appreciated. CWL is also grateful for the encouragement of Prof. Sekerka at the beginning of this study.

\section{References}

[1] W.W. Mullins, R.F. Sekerka, J. Appl. Phys. 35 (1964) 444.

[2] S.R. Coriell, M.R. Cordes, W.J. Boettinger, R.F. Sekerka, J. Crystal Growth 49 (1980) 13.

[3] D.T.J. Hurle, E. Jakeman, A.A. Wheeler, J. Crystal Growth 58 (1982) 163.

[4] G.W. Young, S.H. Davis, Phys. Rev. B 34 (1986) 3388.

[5] S.R. Coriell, G.B. McFadden, J. Crystal Growth 94 (1989) 513.

[6] S.H. Davis, in: D.T.J Hurle (Ed.), Handbook of Crystal Growth 1B: Transport and Stability, North-Holland, Amsterdam, 1994, p. 859.

[7] M.R. Mehrabi, Ph.D. Dissertation, Department of Chemical Engineering, Massachusetts Institute of Technology, Cambridge, Boston, USA, 1994.

[8] R.J. Schaefer, S.R. Coriell, Metall. Trans. 15A (1984) 2109.

[9] N.B. Singh, S.S. Mani, J.D. Adam, S.R. Coriell, M.E. Glicksman, W.M.B. Duval, G.J. Santoro, R. DeWitt, J. Crystal Growth 166 (1996) 364. 
[10] C.W. Lan, F.C. Chen, Comput. Methods Appl. Mech. Eng. 131 (1996) 191.

[11] C.W. Lan, J. Crystal Growth 197 (1999) 983.

[12] R.F. Sekerka, in: P. Hartman (Ed.), Crystal Growth, North-Holland, Amsterdam, 1973, p. 403.

[13] W. Kurz, D.J. Fisher, Fundamentals of Solidification, Trans. Tech. Publications, Brookfields, Vesmonul, 1986.
[14] J. Warren, J.S. Langer, Phys. Rev. E 47 (4) (1993) 2702.

[15] C.W. Lan, M.C. Liang, J.H. Chian, J. Crystal Growth 212 (2000) 340.

[16] P.N. Brown, A.C. Hindmarsh, L.R. Petzold, SIAM J. Sci. Comput. 15 (1994) 1467.

[17] C.W. Lan, M.C. Liang, M.K. Chen, Phys. Fluids 10 (1998) 1329.

[18] L. Nastac, J. Crystal Growth 193 (1998) 271. 